\title{
OPEN The Fuchs corneal dystrophy-associated CTG repeat expansion in the TCF4 gene affects transcription from its alternative promoters
}

\author{
Alex Sirp ${ }^{1,5}$, Kristian Leite $^{1,3,5}$, Jürgen Tuvikene ${ }^{1,2,5}$, Kaja Nurm $^{1}$, Mari Sepp ${ }^{1,4}$ \& \\ Tõnis Timmusk ${ }^{1,2 \bowtie}$
}

The CTG trinucleotide repeat (TNR) expansion in Transcription factor 4 (TCF4) intron 3 is the main cause of Fuchs' endothelial corneal dystrophy (FECD) and may confer an increased risk of developing bipolar disorder (BD). Usage of alternative $5^{\prime}$ exons for transcribing the human TCF4 gene results in numerous TCF4 transcripts which encode for at least $18 \mathrm{~N}$-terminally different protein isoforms that vary in their function and transactivation capability. Here we studied the TCF4 region containing the CTG TNR and characterized the transcription initiation sites of the nearby downstream 5 ' exons 4 a, $4 \mathrm{~b}$ and $4 \mathrm{c}$. We demonstrate that these exons are linked to alternative promoters and show that the CTG TNR expansion decreases the activity of the nearby downstream TCF4 promoters in primary cultured neurons. We confirm this finding using two RNA sequencing (RNA-seq) datasets of corneal endothelium from FECD patients with expanded CTG TNR in the TCF4 gene. Furthermore, we report an increase in the expression of various other TCF4 transcripts in FECD, possibly indicating a compensatory mechanism. We conclude that the CTG TNR affects TCF4 expression in a transcriptspecific manner both in neurons and in the cornea.

Transcription factor 4 (TCF4) is a basic helix-loop-helix transcription factor that plays a vital role in the development of the nervous and immune system ${ }^{1-3}$. TCF4 is expressed in almost every tissue type in human ${ }^{4,5}$. In the brain TCF4 expression peaks during late embryonic development and continues at a relatively high level during postnatal development ${ }^{6,7}$. Notably, transcription from TCF4 gene can begin at multiple mutually exclusive $5^{\prime}$ exons leading to transcripts with varying composition of functional protein domains which modulate the ability of TCF4 to regulate transcription ${ }^{4,8}$.

TCF4 gene is implicated in susceptibility to schizophrenia, and mutations in TCF4 cause Pitt-Hopkins syndrome, a rare developmental disorder characterized by severe motor and mental retardation ${ }^{6,9-11}$. In addition, mutations in the gene regions included only in the longer isoforms of TCF4 have been associated with intellectual disability ${ }^{12}$. Alterations in TCF4 expression levels have been described in patients with depression ${ }^{13}$. An expansion of a CTG TNR, located in an alternative promoter region between TCF4 exons 3 and 4 (known as CTG 18.1), upstream of TCF4 5' exons 4a, 4b and 4c causes Fuchs' endothelial corneal dystrophy (FECD) and has been linked to Bipolar disorder $(\mathrm{BD})^{14,15}$.

FECD is an ocular disorder associated with corneal edema and vision disruption having a varying prevalence between different populations affecting about $4 \%$ of people over 40 in the United States ${ }^{16}$. Out of the many genetic mutations associated with FECD the CTG TNR expansion in TCF4 is thought to be one of the major factors in the development of the disease, as the presence of a TCF4 allele with 50 or more CTG TNR-s confers an increased risk of developing the disease ${ }^{17}$. BD is a psychiatric disorder that affects up to $1 \%$ of the global population, causing severe mood alterations in affected patients ${ }^{18}$. It has been shown that a TCF4 CTG TNR expansion of over 40

\footnotetext{
${ }^{1}$ Department of Chemistry and Biotechnology, Tallinn University of Technology, Akadeemia tee 15, 12618 Tallinn, Estonia. ${ }^{2}$ Protobios LLC, 12618 Tallinn, Estonia. ${ }^{3}$ Present address: Department of Neurology, University Medicine Göttingen, Waldweg 33, 37073 Göttingen, Germany. ${ }^{4}$ Present address: Center for Molecular Biology of Heidelberg University (ZMBH), 69120 Heidelberg, Germany. ${ }^{5}$ These authors contributed equally: Alex Sirp, Kristian Leite and Jürgen Tuvikene. ${ }^{\bowtie}$ email: tonis.timmusk@taltech.ee
} 
A 250 bp TCF4 CTG TNR region

B


E
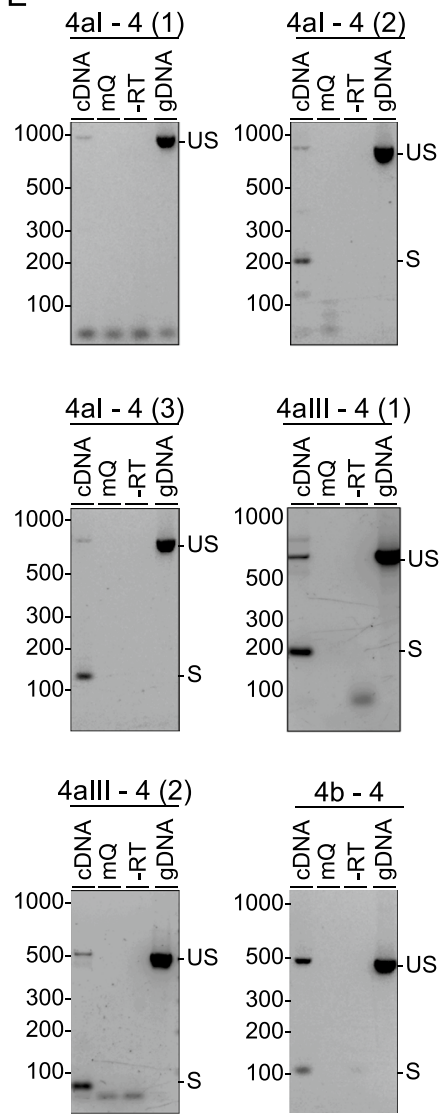
४Figure 1. Transcription start sites, cloned insert sequences and analysis of splice sites in the TCF4 CTG repeatcontaining region in the adult human cerebellum. (A) A schematic of the TCF4 gene including alternative $5^{\prime}$ exons marked as white boxes and internal exons marked as black boxes drawn in scale. Functional protein domains have been marked under the exons and the TCF4 CTG TNR region has been marked on top with an arrow displaying the repeat location in the intron between exons 3 and $4 \mathrm{a}$. Alternative splice sites have been marked as roman numerals. Testis specific exons are underlined and marked bold. (B) Major alternative transcripts of the human TCF4 gene. Coding regions are represented as black boxes and untranslated regions are shown as white boxes. Transcripts are named after the $5^{\prime}$ exon and the splice site. The names of protein isoforms encoded by the transcripts are shown on the right. Locations of alternative splicing that generates fulllength (FL), $\Delta,-$ and + isoforms are shown at the bottom. A and B adapted from Sepp et al. ${ }^{4}$. (C) A schematic drawing of the TCF4 gene region proximal to the CTG TNR. Genomic coordinates are based on the GRCh37/ hg19 genome build. Black boxes indicate internal exons, white boxes 5' exons and striped open box marks the location of the CTG TNR. EST-s from GenBank are shown with accession numbers on the right. TSS peaks (FANTOM5 DPI peak, robust set) and CAGE reads (total counts of CAGE for reverse strand encoding for TCF4) from FANTOM5 project are visualized. (D) Transcripts identified by $5^{\prime}$ RACE are indicated-black boxes show sequenced components, white boxes unsequenced components. Transcription start regions of exon 4aI, exon $4 \mathrm{~b}$ and exon $4 \mathrm{c}$ are indicated by arrows. (E) RT-PCR analysis of the splicing in the region. "US" indicates RT-PCR fragments amplified from gDNA or unspliced pre-mRNA. "S" denotes RT-PCR fragments from spliced mRNA. The numbers in brackets indicate different primer pairs used for PCR. Three different primer pairs were used to identify splice sites between exons $4 \mathrm{a}-\mathrm{I}$ and 4 , whereas two were used to identify splice sites between exons 4a-III and 4. (F) The structure of TCF4 transcripts identified by RT-PCR in D. (G) The cloned promoter regions are indicated with filled boxes and the firefly luciferase (ffLuc) reporter gene are indicated with an open box. $A D$ activation domain, $N L S$ nuclear localization domain, $b H L H$ basic helix-loop-helix, $F L$ full length, $\triangle$ lack of nuclear localization domain, EST expressed sequence tag, TSS transcription start site, FANTOM5 functional annotation of the mammalian genome, DPI decomposition-based peak identification, CAGE cap analysis of gene expression.

CTG TNR-s is frequent in a subset of patients with a severe type of $\mathrm{BD}$ and that the repeat expansion in TCF4 may increase vulnerability to $\mathrm{BD}^{15}$.

Studies on the connection between the TCF4 CTG TNR expansion and the mRNA levels of TCF4 transcripts spanning the TCF4 CTG TNR region have produced contradictory results. A study by Foja et al. ${ }^{19}$ reported that TCF4 CTG TNR expansion is connected with a reduction in the levels of TCF4 transcripts beginning in the proximity of the CTG TNR, whereas a study by Okumura et al. ${ }^{20}$ has reported that TCF4 CTG TNR expansion is connected with an increase in overall TCF4 levels and in the levels of TCF4 transcripts beginning in proximity of the CTG TNR. Two other studies ${ }^{21,22}$ have reported no effect of the CTG TNR expansion on total TCF4 mRNA levels.

It is currently unknown whether the TCF4 CTG TNR expansion affects the levels of TCF4 transcripts and total TCF4 levels. Here, we hypothesized that the TCF4 CTG TNR region contains functional promoters that regulate the transcription of nearby $5^{\prime}$ exons and that the activity of these promoters is altered by the length of TCF4 CTG TNR expansion. For this, we first characterized the TCF4 alternative promoter region containing the CTG TNR by identifying transcription start sites (TSS) and describing the splicing of nearby $5^{\prime}$ exons 4a, $4 \mathrm{~b}$, and $4 \mathrm{c}$. We then used luciferase reporter assay to investigate whether the CTG TNR expansion could influence TCF4 expression in primary neurons by affecting the ability of surrounding regulatory regions to promote transcription. Furthermore, RNA-seq data from corneal tissue of FECD patients with an expanded TCF4 CTG TNR was analyzed to determine the expression levels of TCF4 transcripts beginning both proximal and distal to the CTG TNR. Collectively, our results demonstrate that the CTG TNR expansion differentially modulates the activity of TCF4 promoters.

\section{Results}

Transcription start and splice donor site usage of TCF4 $5^{\prime}$ exons in vicinity of the CTG TNR. The CTG TNR immediately precedes TCF4 $5^{\prime}$ exons $4 \mathrm{a}$, $4 \mathrm{~b}$ and $4 \mathrm{c}$, which are located between internal exons 3 and 4 (Fig. 1A). The major TCF4 transcripts transcribed from the alternative $5^{\prime}$ exons of TCF4 in proximity of the CTG TNR are transcripts encoding for protein isoforms TCF4-B and TCF4-C (Fig. 1B). To characterize the transcription start sites (TSS-s) in this region we performed bioinformatical and 5' RACE analysis. Analysis of GenBank data revealed that a total of 19 expressed sequence tags (EST-s) with 17 different TSS-s can be found between TCF4 internal exons 3 and 4 with none beginning downstream of exon 3 and upstream of the CTG TNR (Fig. 1C). In addition, analysis of TSS peak data from the FANTOM5 (Functional Annotation of the Mammalian Genome) project revealed 6 TSS peaks near the CTG TNR: 1 TSS peak before the CTG TNR and 5 TSS peaks (of which 3 TSS peaks match with TSS-s from GenBank EST-s) downstream of the repeat (Fig. 1C). To validate the potential transcripts and TSS-s from our bioinformatical analysis, we performed $5^{\prime}$ RACE from adult human cerebellar RNA, as it exhibits high levels of TCF4 expression ${ }^{23}$. Our 5' RACE analysis revealed twelve TSS-sthree for exon $4 \mathrm{a}$, three for exon $4 \mathrm{~b}$ and six for exon $4 \mathrm{c}$, distributed across a $\sim 250 \mathrm{bp}$ region (Fig. 1D). Out of the 12 TSS-s detected by $5^{\prime}$ RACE only 4 matched with the TSS-s from our bioinformatical analysis. Importantly, the CTG TNR was never present in the 5' UTR of exon 4a, 4b and 4c transcripts since EST-s from GenBank and our 5' RACE revealed no TSS directly upstream of the TCF4 CTG TNR region (Fig. 1D, Supplementary Fig. S1). When considering previously published data about the TSS-s of TCF4 exon 4a, 4b and 4c and data obtained from our 5' RACE analysis, the promoters in the TCF4 CTG TNR region appear to be dispersed promoters, 
A

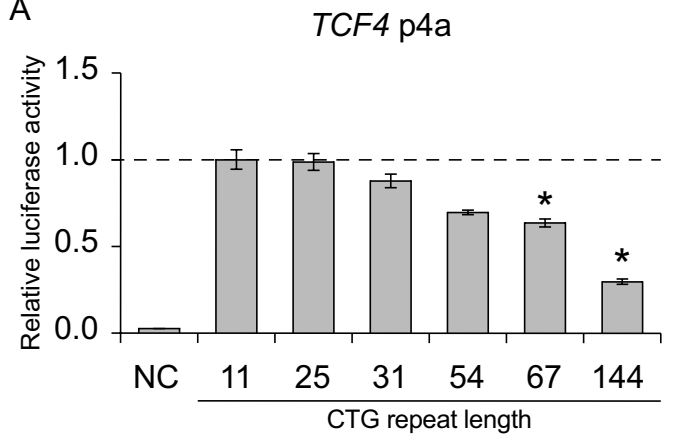

B

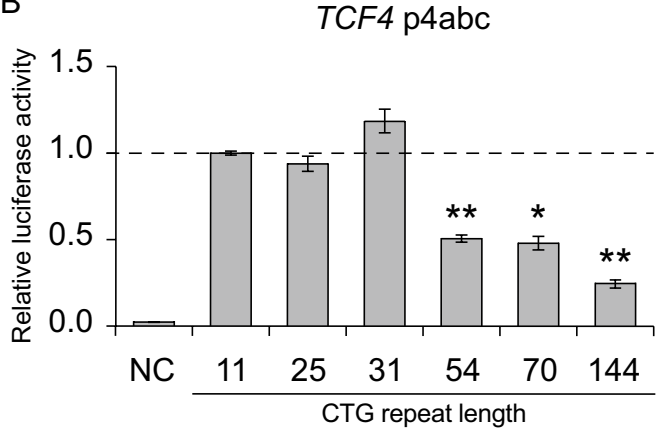

Figure 2. The TCF4 CTG trinucleotide repeat region modulates the activity of proximal downstream TCF4 promoters in a length dependent manner in neurons. (A,B) Promoter activities of TCF4 p4a (A) and TCF4 p4abc (B) constructs with different CTG repeat sizes $(11,25,31,54,67$ or 70,144$)$. Promoter activity was measured in rat cultured cortical neurons transfected with luciferase reporter constructs. Three independent experiments were performed for each promoter construct. A negative control (NC, promoterless pGL4.15 vector) is also shown. Statistical analysis was performed using one-way repeated-measures analysis of variance (ANOVA) with Greenhouse-Geisser correction followed by Dunnett's post hoc test. Statistical significance is shown compared to the construct with $11 \mathrm{CTG}$ repeats $\left({ }^{\star} \mathrm{p}<0.05,{ }^{\star *} \mathrm{p}<0.01\right)$. Luciferase activity is shown relative to the luciferase signal obtained with the respective promoter construct with $11 \mathrm{CTG}$ repeats.

which are defined as a type of promoter where transcription start sites are spread across a region of around 100 nucleotides $^{24-26}$.

To study the usage of splice donor sites at the 5' exons located near the CTG TNR, we amplified the fragments encompassing the splice sites from adult human cerebellar RNA using RT-PCR. We identified all previously described TCF4 exon $4 \mathrm{a}$ and $4 \mathrm{~b}$ splice sites ${ }^{4}$ in adult human cerebellum but could not detect mRNAs starting with exon 4a-II. Similar results were obtained in our previous study ${ }^{4}$, although one respective sequence is present in GenBank, suggesting that the levels of these TCF4 transcripts are very low. In addition, we found that during splicing, donor site closest to the TSS is used. For instance, TCF4 mRNAs initiated upstream of 4a-I splice site used donor site $4 \mathrm{a}-\mathrm{I}$ exclusively and not downstream splice sites $4 \mathrm{a}-\mathrm{III}$ or $4 \mathrm{~b}$ (Fig. 1D-F). The RT-PCR analysis confirmed the absence of TSS-s directly upstream of CTG TNR (Fig. 1E). Collectively these results revealed that TCF4 CTG TNR is not included in the $5^{\prime}$ UTR of exon $4 \mathrm{a}, 4 \mathrm{~b}$ and $4 \mathrm{c}$ transcripts and instead locates in a dispersed promoter $^{24-26}$ region characterized by spread TSS distribution.

Activity of TCF4 promoters immediately downstream of the CTG TNR decreases with increasing repeat length. We next determined whether the region surrounding the CTG TNR in TCF4 intron 3 upstream of alternative $5^{\prime}$ exons contains functional promoters (Fig. 1C). For that, we analyzed two DNA fragments-a shorter sequence (TCF4 p4a) spanning the TCF4 CTG TNR region from just downstream of exon 3 into $5^{\prime}$ exon $4 \mathrm{a}$ and a longer sequence (TCF4 p4abc) spanning the entire TCF4 CTG TNR from just downstream of exon 3 to inside exon 4 (Fig. 1G). We cloned these fragments into pGL4.15[luc2P/Hygro] luciferase reporter vectors and transfected the vectors into rat primary cortical neurons. The expression of luciferase was increased by $>30$-fold using reporter constructs containing either $\mathrm{p} 4 \mathrm{a}$ or $\mathrm{p} 4 \mathrm{abc}$ sequences when compared to a negative control construct without a promoter (Fig. 2A,B). These results indicate that $\mathrm{p} 4 \mathrm{a}$ and $\mathrm{p} 4 \mathrm{abc}$ sequences contain functional promoters.

To assess the effect of the CTG TNR length on the activity of TCF4 p4a and p4abc promoter regions, we generated twelve luciferase reporter constructs where each construct contained either the TCF4 p4a or p4abc promoter sequence combined with six different CTG TNR lengths (11, 25, 31, 54, 67/70, 144). The luciferase reporter assay revealed that an extended CTG TNR with a length of 54, 67/70 or 144 repeats significantly reduced the activity of the promoter for both TCF4 p4a and p4abc (Fig. 2A,B). The presence of 144 repeats reduced the activity of $\mathrm{p} 4 \mathrm{a}$ and $\mathrm{p} 4 \mathrm{abc}$ by $70 \%(\mathrm{p}=0.0192)$ and $75 \%(\mathrm{p}=0.0095)$, respectively. These results demonstrate that the CTG TNR expansion interferes with transcription from the TCF4 p4abc promoter region.

The CTG TNR expansion in TCF4 gene affects the transcription of TCF4 alternative $5^{\prime}$ exons in FECD patients. To describe whether an increased CTG TNR affects the expression of different TCF4 transcripts, we performed a comprehensive analysis of two previously published RNA-seq datasets from the corneal endothelium of FECD patients with an expanded TCF4 CTG TNR and control groups without the repeat expansion $^{27,28}$. The 2019 dataset generated by Nikitina et al. includes 6 controls and 8 FECD patients with an expanded TCF4 CTG TNR ${ }^{27}$, and the 2020 dataset by Chu et al. includes 9 controls and 6 FECD patients with an expanded TCF4 CTG TNR ${ }^{28}$. First, we evaluated how the levels of transcripts beginning from the CTG TNR region change in FECD. The expression levels of exons 4aI and 4aIII showed a strong decrease in FECD patients, which is in agreement with our luciferase reporter assays in neurons (Fig. 3). In contrast, the levels of transcripts containing $5^{\prime}$ exon 4c were increased in FECD patients (Fig. 3). 


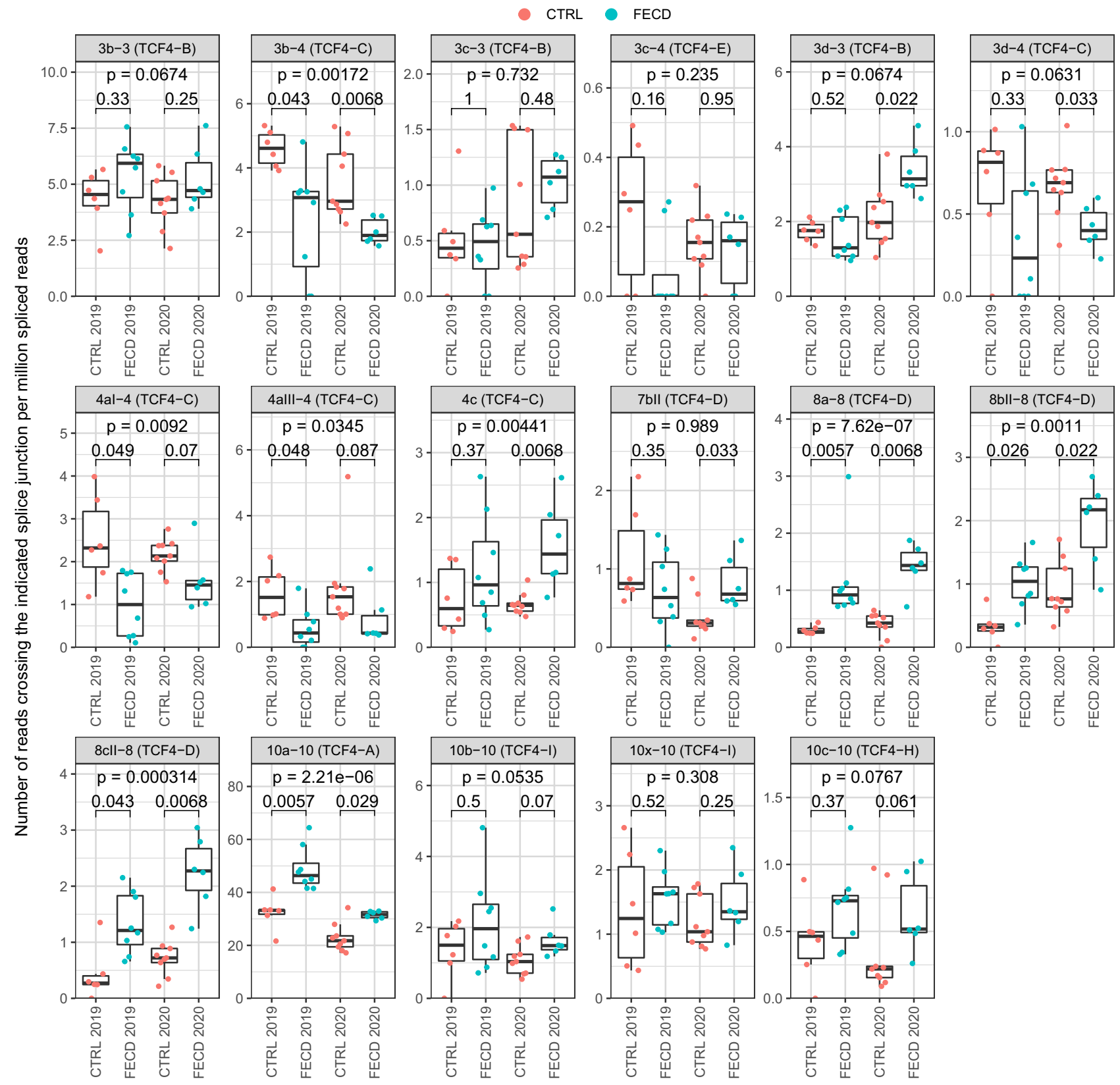

Figure 3. The expression of TCF4 alternative transcripts containing $5^{\prime}$ exons spliced to exon 4 is decreased in the cornea of FECD patients. Two independent FECD RNA-seq datasets-by Nikitina et al. ${ }^{27}$ (named 2019 in the figure) and Chu et al..$^{28}$ (named 2020 in the figure)-were used to analyze the expression levels of TCF4 alternative $5^{\prime}$ exons in corneal endothelium. The expression levels of different TCF4 splicing events were quantified using the number of reads crossing the splice junction (shown above the graphs) normalized with the total number of spliced reads and multiplied by million. The TCF4 protein isoform encoded by the transcripts containing the indicated splicing event is shown in parentheses above the graphs. The data is visualized as box plots-the hinges show $25 \%$ and $75 \%$ quartiles, the horizontal line shows the median value, the upper whisker extends from the hinge to the largest value no further than $1.5^{*}$ inter-quartile range from the hinge, the lower whisker extends from the hinge to the smallest value at most $1.5 *$ inter-quartile range of the hinge. All data points are shown with dots. The 2019 dataset includes 6 controls and 8 FECD patients with an expanded TCF4 CTG TNR, and the 2020 dataset includes 9 controls and 6 FECD patients with an expanded TCF4 CTG TNR. $10 \times-10$ is a previously undescribed splicing event. Within-experiment statistical analysis between the CTRL and FECD patients (indicated groups) was done using Mann-Whitney U test, $p$-values were corrected for multiple testing using false discovery rate. Generalized linear model, followed by Wald test was used to determine statistical significance of the disease state combining data from both 2019 and 2020 experiments, p-values were corrected using FDR (p-value in the upper part of the panel). 
Next, we investigated whether the CTG TNR affects the levels of TCF4 transcripts starting from far upstream of the CTG TNR (e.g. exons 3b, 3c, etc.). Our analysis revealed that FECD patients with an expanded CTG TNR displayed reduced levels of transcripts containing TCF4 alternative $5^{\prime}$ exons $3 \mathrm{~b}$ and $3 \mathrm{~d}$ spliced directly to internal exon 4 , just downstream of the repeat region, thus skipping internal exon 3 (Fig. 3). In contrast, the levels of transcripts containing these exons spliced to the internal exon 3 were either not changed (exons $3 \mathrm{c}$ and $3 \mathrm{~d}$ ) or were upregulated (exon $3 b$ ) in FECD. These results suggest that the CTG TNR affects both promoter activity and alternative splicing in transcripts starting from upstream of the repeat region (Fig. 3). Notably, we also found that FECD patients had increased levels of transcripts containing $5^{\prime}$ exons $8 \mathrm{a}, 8 \mathrm{bII}, 8 \mathrm{cII}$ and $10 \mathrm{a}$, which are all located far downstream of the CTG TNR (Fig. 3).

Different TCF4 transcripts encode for various TCF4 protein isoforms (Fig. 1B) that vary in their function and transactivation capability ${ }^{4,8}$. The major TCF4 transcripts comprising of $5^{\prime}$ exons $3 \mathrm{~b}, 3 \mathrm{c}$ and $3 \mathrm{~d}$ encode for isoform TCF4-B when spliced to internal exon 3; transcripts with exons $3 \mathrm{~b}$ and $3 \mathrm{~d}$ encode for isoform TCF4-C when spliced to exon 4; transcripts with exons $8 \mathrm{a}$, $8 \mathrm{bII}$ and $8 \mathrm{cII}$ encode for isoform TCF4-D when spliced to exon 8; transcripts with exons $10 \mathrm{a}, 10 \mathrm{~b}$ and $10 \mathrm{c}$ spliced to exon 10 encode for isoforms TCF4-A, TCF4-I and TCF4-H, respectively (Fig. 1B). Data from TCF4 transcripts which encode the same protein isoform were combined to determine whether the levels of different TCF4 transcripts encoding specific TCF4 protein isoforms are changed in FECD. We found that the expression of transcripts encoding isoform TCF4-C decreased while the levels of isoforms TCF4-A, TCF4-B, TCF4-D and TCF4-H increased in FECD (Fig. 4). These observations were confirmed by analyzing TCF4 transcripts by the expression levels of internal exons. Decreases in transcripts comprising of internal exons 6-8 were observed in FECD patients, which can be explained by reduced expression of isoform TCF4-C encoding mRNAs (Figs. 4, 5). FECD patients and the control group exhibited equal amounts of transcripts containing exons 8 and 9 (Fig. 4). The sudden elevation of transcripts comprising of exons 8 and 9 when compared to exons 4-8 in FECD patients accounts for the increase in the expression of isoform TCF4D encoding mRNAs (Figs. 4,5). An increase in the levels of transcripts containing exons 10-16 present in all TCF4 transcripts is caused by the increase in the expression of TCF4-A, TCF4-B and TCF4-D mRNAs in FECD patients (Fig. 4). As a contradictory result we saw that transcripts containing exons 3 and 4 which should reflect the levels of isoform TCF4-B did not increase in FECD patients even though the levels of transcripts containing 5 ' exons included in isoform TCF4-B did increase (Fig. 3).

Only the major transcripts/splicing events are reported in the Figs. 3, 4 and 5. The results of all studied TCF4 exons/splicing events and isoforms can be found in Supplementary Fig. S2. In conclusion, the expression levels of TCF4 transcripts were altered in FECD patients - the repeat expansion caused a reduction in transcripts starting immediately downstream of CTG TNR and transcripts containing $5^{\prime}$ exons spliced directly to exon 4 , and an increase in transcripts encoded by distal 5' exons located hundreds of kbp downstream of the repeat. The results of RNA-seq experiments have been summarized in Fig. 5.

\section{Discussion}

Previous studies have indicated that the CTG TNR expansion in intron 3 of TCF4 strongly increases the risk of developing FECD and also vulnerability and severity of $\mathrm{BD}^{15,29}$. The pathogenic mechanism of the TCF4 CTG TNR and other TNR-s in general is still a major question. We investigated the hypothesis that the CTG TNR impacts the transcription of TCF 4 mRNAs initiated from nearby $5^{\prime}$ exons, leading to an imbalance of the levels of alternative TCF4 protein isoforms. We show for the first time that the expansion of the CTG TNR directly reduces the activity of the nearby downstream TCF4 promoters in a length dependent manner-longer, more expanded repeats reduce the activity of proximal downstream promoters. The lengths of the extended repeats that were studied fit into the pathogenic range for both bipolar disorder $(>40)$ and Fuchs' dystrophy $(>50)^{14,15}$. Soliman et al. has shown that the severity of FECD correlates with the length of the CTG TNR in TCF4 as patients with a CTG TNR expansion exhibited a more severe form of FECD, but the mechanism underlying this phenotype remains unknown ${ }^{30}$. It is important to note that our determination of TSS-s by $5^{\prime}$ RACE and reporter experiments were done using human cerebellar RNA and rat cultured cortical neurons, respectively. Therefore, it would be interesting to conduct similar experiments in human corneal endothelial cells. This would help to translate our findings between different cell types and further validate the effect of the CTG TNR expansion on transcription also in FECD patients.

Detailed analysis of previously published FECD RNA-seq datasets ${ }^{27,28}$ revealed that the levels of TCF4 transcripts containing alternative $5^{\prime}$ exons $4 \mathrm{aI}$ and $4 \mathrm{aIII}$ were reduced in the corneal endothelial cells of FECD patients with an expanded CTG TNR. These results support our findings that the TCF4 CTG TNR expansion reduces the activity of proximal downstream promoters linked to these $5^{\prime}$ exons also in human corneal endothelium. Interestingly, an increase in the levels of TCF4 transcripts encoded by downstream alternative $5^{\prime}$ exons distal to the CTG TNR was also noted, which may indicate a compensatory mechanism to rescue the levels of TCF4 protein arising from the deficit of transcripts encoding TCF4-C. This compensation phenomenon needs to be considered when studying TCF4 expression levels in FECD and other diseases connected with the TCF4 intronic CTG TNR and could explain why different research groups have published contradictory results concerning changes in TCF4 levels when studying FECD ${ }^{19-22}$. Our results indicate that the levels of TCF4 transcripts change bidirectionally in response to an expanded CTG TNR - transcripts beginning near the repeat region decline just as Foja et al. reported ${ }^{19}$ while certain transcripts beginning downstream of the repeat region increase as reported here, and mask the decrease of long TCF4 transcripts. As the expression of different TCF4 transcripts decline and rise simultaneously the overall TCF4 levels may not change significantly in FECD as has been reported by Mootha et al. ${ }^{21}$ and Ołdak et al. ${ }^{22}$. In contrast, Okumura et al. ${ }^{20}$ found an increase in total TCF4 expression levels which is also evident in our RNA-seq analysis as we saw a slight rise in TCF4 expression when measuring the expression of internal exons present in all TCF4 transcripts (exons 10-21). Our results illustrate the importance 
A

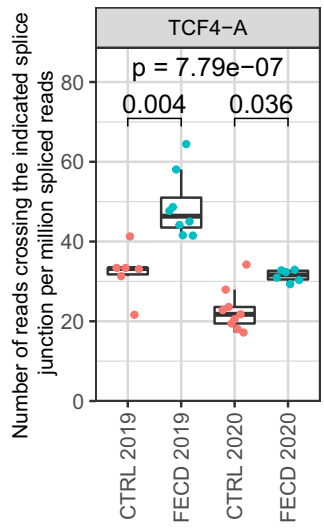

B

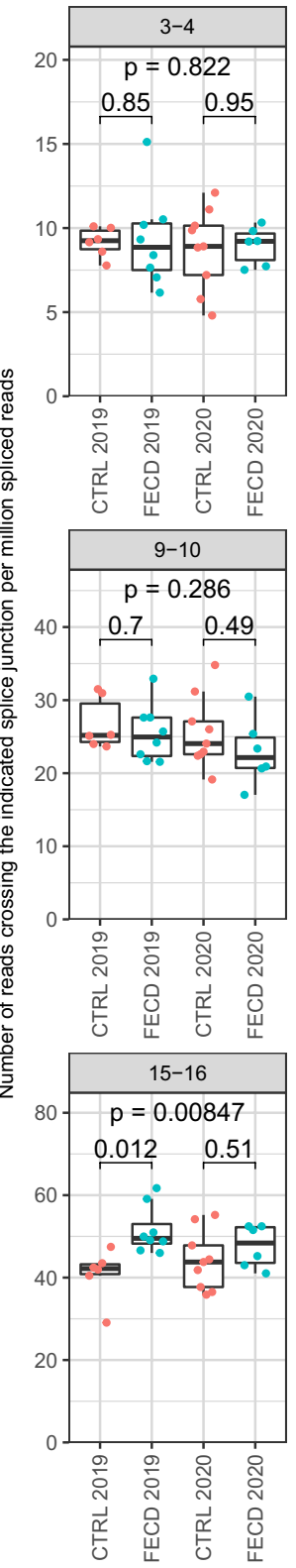

- CTRL


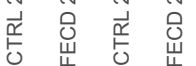
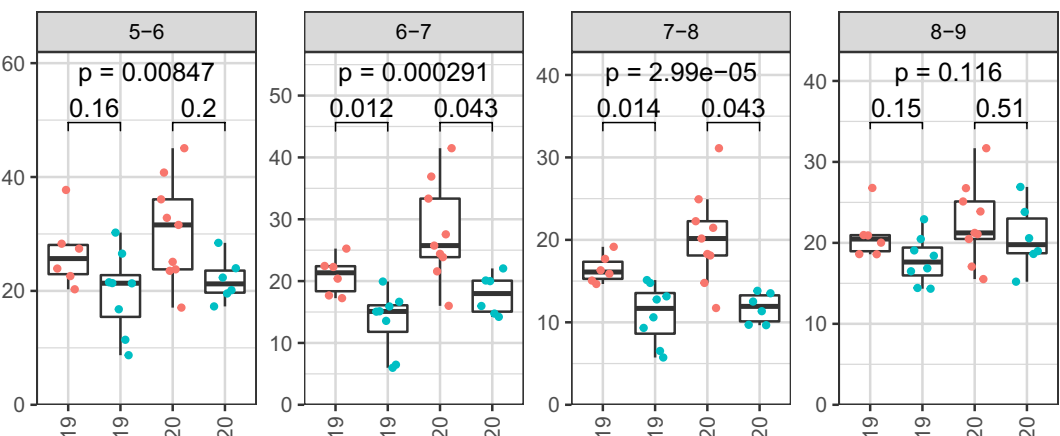

$10-$



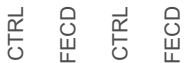

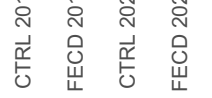

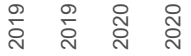

旁
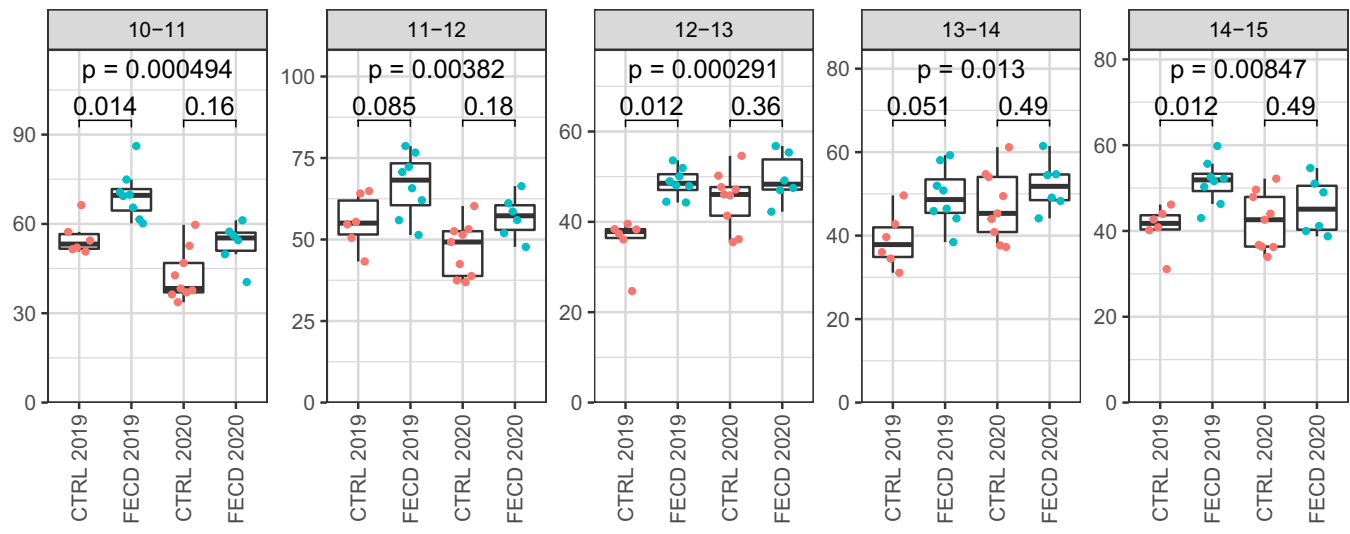

.
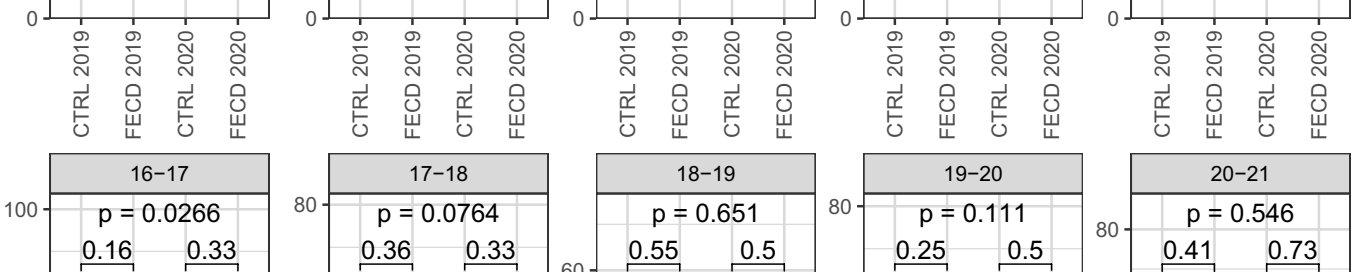

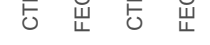
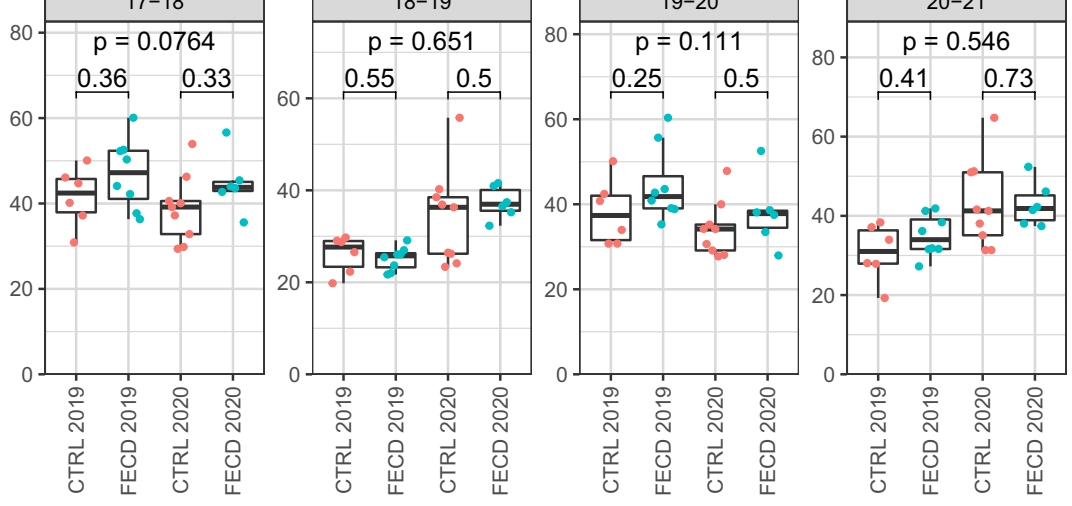

Figure 4. Expression of transcripts encoding TCF4-C isoform are downregulated, whereas transcripts encoding other TCF4 isoforms are upregulated in the cornea of FECD patients. Two independent FECD RNA-seq datasets-by Nikitina et al. ${ }^{27}$ (named 2019 in the figure) and Chu et al. ${ }^{28}$ (named 2020 in the figure)-were used to analyze the expression levels of TCF4 exons in corneal endothelium. The expression levels of (A) TCF4 isoforms and (B) internal exons were quantified using the number of reads (A) from transcripts encoding for the respective isoform (shown above the graphs) and (B) crossing the indicated splice junction (shown above the graphs). Data was normalized with the total number of spliced reads and multiplied by million. For more details, see legend of Fig. 3. 


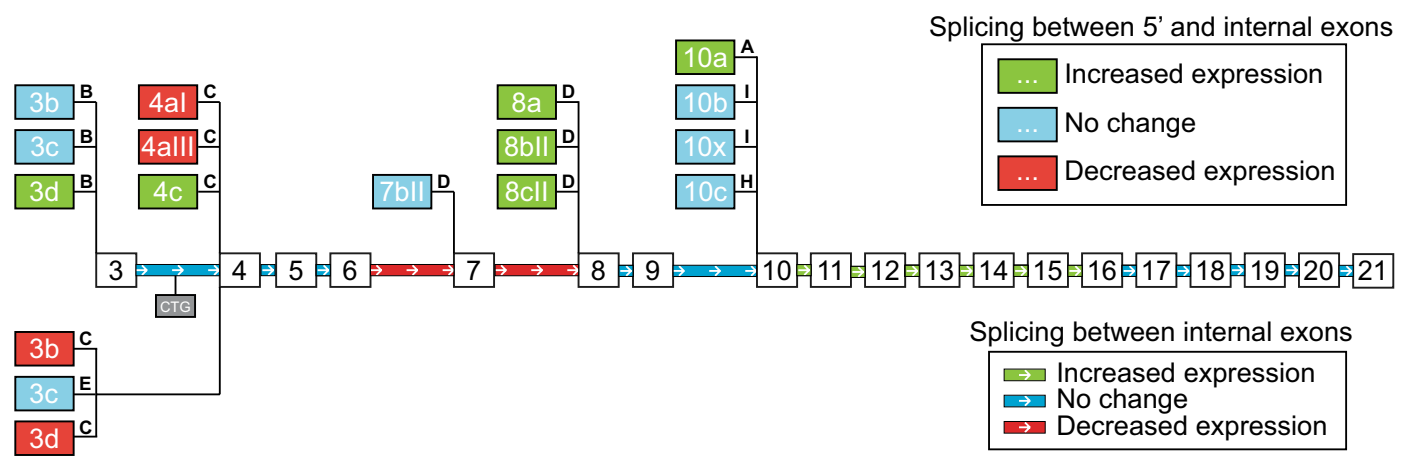

Figure 5. Summary of the expression levels of TCF4 alternative $5^{\prime}$ and internal exons in FECD. Schematic depiction of the human TCF4 gene structure and transcripts which displayed high expression levels in RNA-seq data analysis (see Figs. 4, 5). Internal exons are depicted as boxes (3-21) and 5' exons are shown as columns on top or under the internal exons. Name of the respective TCF4 exon is shown inside the box. Splicing of $5^{\prime}$ exons is shown by black lines which lead to the respective internal exon. Isoform encoded by the transcript is indicated as a single character (A, B, C, D, E, H or I) marked bold on the line next to the 5' exon. 5' exons marked green or red display significantly increased or decreased expression, respectively, in FECD, when spliced to the respective internal exon. Internal exons are connected by green, blue or red lines. A green or a red line between internal exons indicates a significant increase or decrease, respectively, in the expression level of transcripts containing splicing between these exons in FECD. A blue line or box indicates no change in the levels of transcripts containing splicing between these exons. The CTG TNR is marked between internal exons 3 and 4 as a grey box (CTG).

of the exact transcript measured when studying TCF4 expression levels. The original RNA-seq study by Chu and others concluded that the TCF4 CTG TNR expansion increases the stability and thus the amount of expanded CTG repeat-including intronic RNAs in the corneal endothelium and causes comprehensive changes in splicing. No alterations in the overall expression of mature TCF 4 mRNA was noted ${ }^{28}$. The study by Nikitina et al. was a data article and no conclusions were made ${ }^{27}$.

Interestingly, we also detected an increase in the expression of 5' exons spliced to exon 3 encoding for TCF4B in patients with FECD, showing that almost all the TCF4 promoters far upstream from the CTG TNR had increased activity due to the repeat expansion. However, the increase in upstream promoter activity did not reflect in the levels of transcripts containing exons 3 and 4. It is plausible that the CTG repeat expansion could regulate transcriptional elongation of RNA polymerase by slowing down the polymerase in the CTG TNR region ${ }^{31}$. This can cause an accumulation of RNA polymerases in the CTG TNR and dissociation of the polymerase, leading to a decrease of full-length TCF4 transcripts beginning from upstream of the CTG repeat. An increase in the expression of $5^{\prime}$ exons spliced to exon 3 may be due to preferential splicing of transcripts to the exon before the CTG TNR (exon 3), as changes in splicing have been described before in diseases associated with repeat expansion ${ }^{31}$.

We have previously shown that TCF4 protein isoforms can be divided into longer and shorter isoforms which vary in their function and transactivation capability ${ }^{4,8}$. Currently FECD research focuses mainly on the CTG TNR and missplicing of longer TCF4 transcripts in FECD ${ }^{17}$, and little research has been done to analyze the expression of all the TCF4 transcripts in FECD. Our detailed analysis provides new insight into FECD as we show that the CTG TNR directly modulates the expression of TCF4 which may be among the underlying causes for the development of the disease. Since TCF4 mRNAs detected in the present study are expressed virtually in all tissues, with high levels in the fetal and postnatal brain ${ }^{4}$, there may also be a similar correlation between the CTG TNR length and the expression levels of TCF4 transcripts in vivo in the brain which could predispose development of BD. However, it should be noted that the link between the CTG TNR expansion in TCF4 and $\mathrm{BD}$ has only been shown once and has not been reported by newer studies.

Strong evidence has also been provided in support of a mechanism in which the toxic (CUG) $)_{n}$ TNR containing TCF4 $m$ RNAs are the cause of Fuchs' corneal dystrophy ${ }^{16,32,33}$. According to this mechanism, the TNRcarrying RNAs cluster RNA binding proteins, interfering with the splicing of various mRNAs. Of note, antisense therapy using Fuchs' dystrophy ex vivo cell models leads to inhibition of RNA foci and mis-splicing in Fuchs' dystrophy ${ }^{34,35}$. Since the CTG TNR is located in the intron between exons 3 and 4 , this repeat is not included in the fully mature TCF4 mRNA m $^{4}$ but the CTG TNR is still included in the pre-mRNA of transcripts initiated at the upstream promoters (exon 1a, 1b, 3a, 3b, 3c, 3d promoters).

Repeat expansions have been associated with more than 40 diseases ${ }^{29}$ and unstable TNR-s may occur in both coding and noncoding regions, including promoters, introns and untranslated regions (UTR) of genes ${ }^{36}$. Among noncoding TNR-s, one of the most studied is the TNR repeat (CGG) located in the 5' UTR of Fragile X Mental Retardation (FMR1) gene. This TNR causes hypermethylation and silencing or increases in the expression level of the gene, depending on TNR length ${ }^{37}$. TNR diseases with TNR in the promoter region of the affected gene have been less studied. Recently, an intronic polymorphic CGG repeat in a conserved alternative promoter of the AFF3 gene, an autosomal homolog of the X-linked AFF2/FMR2 gene, was shown to lead to hypermethylation of the promoter and transcriptional silencing of AFF3 expression in the brain ${ }^{38}$. However, the effect of TNR on promoter activity using transient expression analysis of promoters linked to TNR was not studied. Research on 
Friedreich ataxia, which is caused by an expansion of the intronic TNR (GAA) in the FXN gene, has revealed reduced expression of the gene in patient derived cell lines ${ }^{39}$. A hexamer repeat expansion (GGGGCC) located in the $5^{\prime}$ regulatory region of the C9ORF72 gene, causing hereditary amyotrophic lateral sclerosis, has been shown to reduce the ability of the surrounding region to promote the expression of a reporter protein in human kidney and neuroblastoma cell lines ${ }^{40}$. Overall, these results indicate that expansion of TNR can alter the expression of the nearby genes. This is in agreement with our results showing that the expression levels of different TCF4 transcripts are altered in FECD due to the CTG TNR expansion.

Taken together, our results help to explain why previous research on the levels of TCF4 transcripts in FECD has displayed varying results. Analyzing only total TCF4 levels or levels of certain TCF4 transcripts can produce misleading results due to the complexity of the TCF4 gene and its regulation. The current study shows that the TCF4 CTG trinucleotide repeat expansion modulates the activity of nearby TCF4 promoters in a length dependent manner-an expanded CTG TNR causes reduction in promoter activity. Analysis of RNA-seq datasets revealed that the expression levels of the many TCF4 transcripts are increased or decreased simultaneously in the cornea of FECD patients. Further work is needed to elucidate the exact mechanism how this repeat region affects TCF4 transcription and whether the changed TCF4 levels contribute to the development of FECD and BD.

\section{Methods}

Generation of DNA constructs. Human postmortem tissues were used to obtain DNA and RNA samples. All protocols using human tissue samples were approved by Tallinn Committee for Medical Studies, National Institute for Health Development (Permit Number 402). All experiments were performed in accordance with relevant guidelines and regulations.

TCF4 gene fragments were screened from human DNA samples for the TCF4 CTG TNR length and fragments with the desired CTG TNR length were amplified by PCR from $20 \mathrm{ng}$ of genomic DNA in a $20 \mu \mathrm{l}$ mixture using 0.4 units of Phusion Hot Start II (Thermo Scientific) and primer p4a_p4abc_F paired with the p4abc_R or p4a_R primer (Supplementary Table S1) with a final concentration of $0.25 \mu \mathrm{M}$ to amplify the longer (TCF4 p4abc) and the shorter (TCF4 p4a) sequence of the TCF4 gene (Fig. 2). Following amplification, the PCR mixtures were incubated for $15 \mathrm{~min}$ at $72{ }^{\circ} \mathrm{C}$ with 1 unit of FirePol DNA polymerase (Solis BioDyne) for the synthesis of adenosine overhangs for cloning. The PCR products were first inserted into the pSTBlue-1 acceptor vector (Merck Millipore) and then to the pGL4.15[luc2P/Hygro] luciferase reporter vector (\#E6701, Promega).

Promoter regions encompassing CTG TNR-s with five different lengths $(11,25,31,54$ and 67 or 70 repeats) were acquired from human genomic DNA by PCR. A sixth synthetic DNA segment with 144 CTG repeats was ordered from GenScript. All the generated constructs were verified by sequencing as the length of TNR tended to be unstable in bacteria when producing plasmids (Supplementary Table S1).

Luciferase reporter assay and neuron cultures. The protocols involving animals were approved by the ethics committee of animal experiments at Ministry of Agriculture of Estonia (Permit Number: 45). All experiments were performed in accordance with the relevant guidelines and regulations.

Prenatal rat cortical neurons were cultured as described previously ${ }^{41}$. Neurons grown 6 days in vitro were transfected with $180 \mathrm{ng}$ firefly reporter construct and $20 \mathrm{ng}$ pGL4.83[hRlucP/PGK1/Puro] as described previously ${ }^{4}$ for $4 \mathrm{~h}$ on a plate shaker using Lipofectamine 2000 (\#11668019, Thermo Fisher Scientific) with a reagent to DNA ratio 3:1. Two days after transfection neurons were lysed in $50 \mu$ l Passive Lysis Buffer (Promega) and luciferase reporter assay was performed using the Dual-Glo Luciferase Assay System (Promega) according to manufacturer's protocol. Luciferase signals were measured using the GENios Pro microtiter plate reader (Tecan). For analysis, the signals were first normalized to the signal of the Renilla luciferase and then normalized to the respective ratio in cells transfected with the 11 repeat CTG construct. One-way repeated-measures analysis of variance (ANOVA) with Greenhouse-Geisser correction followed by Dunnett's post hoc test was used to determine the statistical significance compared to the luciferase signals from the 11 repeat CTG construct group.

$5^{\prime}$ rapid amplification of DNA ends (5'RACE) analysis and reverse transcription polymerase chain reaction (RT-PCR). Total RNA from post-mortem adult human cerebellum was treated with Turbo DNase (Thermo Fisher Scientific) according to the supplier's protocol. 5' RACE analysis was carried out on human cerebellar RNA using the GeneRacer Kit (Thermo Fisher Scientific) according to manufacturer's protocol with primers outlined in Supplementary Tables S1 and S2.

For RT-PCR, cDNA was synthesized from human cerebellar RNA using 100 units of SuperScript III reverse transcriptase (Thermo Fisher Scientific) with oligo $(\mathrm{dT})_{20}$ and a random hexamer primer mixture (1:1 ratio, Microsynth) according to the manufacturer's protocol. A negative control (-RT) was also included where Superscript III reverse transcriptase was not added. After cDNA synthesis, PCR was performed in $20 \mu \mathrm{l}$ using 3 units of Hot FirePol (Solis Biodyne) and primers listed in Supplementary Table S1 with a final concentration of $0.25 \mu \mathrm{M}$. All the sense primers used for RT-PCR were combined with the antisense primer hTCF4_exon4_as2 except for sense primer hTCF4_4aIII_s (2) which was used together with the antisense primer hTCF4_exon4_as1.

Bioinformatic analysis. Cap Analysis of Gene Expression (CAGE) data from the Functional Annotation of the Mammalian Genome project phase 5 (FANTOM5) ${ }^{42}$ was used to locate potential TCF4 TSS-s. Both predicted TSS-s (FANTOM5 DPI, robust set) and total counts of CAGE reads for the reverse strand (encoding for TCF4) were visualized in UCSC Genome Browser together with the EST-s from GenBank (accessed at 10.07.2020) in the area surrounding the TCF4 CTG TNR region (chr18:53,254,500-53,252,500, human GRCh37/hg19 assembly). The FANTOM5 data can be accessed at https://fantom.gsc.riken.jp/5/datahub/hg19/ reads/ctssTotalCounts.rev.bw. 
Raw RNA-seq data from corneal endothelium of FECD patients and controls (see Supplementary Table S3 for sample information) were obtained from Sequence Read Archive database (accession numbers PRJNA524323 ${ }^{27}$ and SRP238609 ${ }^{28}$ ) using prefetch tool (version 2.10.0) from the SRA toolkit. Reads in fastq format were extracted using fasterq-dump. Adapter and quality trimming was done using BBDuk (part of bbmap version 38.79) using the following parameters: $\mathrm{ktrim}=\mathrm{rk}=23 \mathrm{mink}=11$ hdist $=1$ tbo qtrim $=1 \mathrm{r}$ trimq $=10$ minlen $=100(\operatorname{minlen}=85$ for data from PRJNA524323). Reads were mapped to hg19 genome (primary assembly and annotation obtained from GENCODE, release 34, GRCh37) using STAR aligner (version 2.7.3a) with default parameters. To increase sensitivity for unannotated splice junctions, splice junctions obtained from the 1st pass were combined (per dataset) and filtered as follows: junctions on mitochondrial DNA and non-canonical intron motifs were removed; only junctions supported by at least 6 reads in the whole dataset were kept. The filtered junctions were added to the 2nd pass mapping using STAR. Intron-spanning reads were quantified using FeatureCounts (version 2.0.0) with the following parameters: - $\mathrm{p}-\mathrm{B}-\mathrm{C}-\mathrm{s} 2$-J. To count reads from TCF4 extended exons (exons $4 \mathrm{c}$ and $7 \mathrm{bII}$ ), reads crossing a region $2 \mathrm{bp} 5^{\prime}$ from the internal exon (exon 4 and 7 , respectively) were quantified using FeatureCounts and a custom-made saf file. Splice junctions in the TCF4 region showing less than 4 reads for the whole dataset were discarded, the rest of the splice junctions associated with TCF4 were manually curated and annotated according to Sepp et al. ${ }^{4}$. A custom R script was used to quantify the expression of different TCF4 splice variants. Reads crossing the indicated splice junctions were normalized using the number of all splice-junction crossing reads in the respective samples. Then, data summed by the Exon column (see Supplementary Table S4) to obtain expression levels of splice junctions for TCF4 5' exons. Next, data was aggregated by the Isoform column (see Supplementary Table S4) to obtain expression levels of spliced reads of TCF4 internal exons and transcripts encoding different TCF4 protein isoforms. The annotated splice junction table for quantifying different TCF4 splice sites and transcripts encoding different isoforms is shown in Supplementary Table S4. The results were visualized using ggplot2 package (version 3.3.1) in R (version 4.0.1). Statistical analysis of the RNA-seq data was carried out in R as follows. To determine statistical significance between control and FECD patients within an experiment, non-parametric Mann-Whitney U-test was performed, $\mathrm{p}$-values were corrected for multiple comparisons within experiment (per figure) using false discovery rate (FDR). To determine general statistical significance of the disease state for combined data of the two experiments, normalized data was transformed by adding 0.01 , followed by fitting generalized linear model with Gamma distribution using Experiment + Disease + Experiment:Disease as the model. p-value for the disease state was obtained using Wald test and corrected for multiple comparisons using FDR (per figure).

Received: 17 August 2020; Accepted: 14 October 2020

Published online: 28 October 2020

\section{References}

1. Zhuang, Y., Cheng, P. \& Weintraub, H. B-lymphocyte development is regulated by the combined dosage of three basic helix-loophelix genes, E2A, E2-2, and HEB. Mol. Cell. Biol. 16, 2898-2905 (1996).

2. Guillemot, F. Spatial and temporal specification of neural fates by transcription factor codes. Development 134, 3771-3780 (2007).

3. Zweier, C. et al. Haploinsufficiency of TCF4 causes syndromal mental retardation with intermittent hyperventilation (Pitt-Hopkins syndrome). Am. J. Hum. Genet. 80, 994-1001 (2007).

4. Sepp, M., Kannike, K., Eesmaa, A., Urb, M. \& Timmusk, T. Functional diversity of human basic helix-loop-helix transcription factor TCF4 isoforms generated by alternative 5' exon usage and splicing. PLoS ONE 6, e22138 (2011).

5. Fagerberg, L. et al. Analysis of the human tissue-specific expression by genome-wide integration of transcriptomics and antibodybased proteomics. Mol. Cell Proteomics 13, 397-406 (2014).

6. Rannals, M. D. \& Maher, B. J. Molecular mechanisms of transcription factor 4 in Pitt Hopkins syndrome. Curr. Genet. Med. Rep. 5, 1-7 (2017).

7. Ma, C., Gu, C., Huo, Y., Li, X. \& Luo, X.-J. The integrated landscape of causal genes and pathways in schizophrenia. Transl. Psychiatry https://doi.org/10.1038/s41398-018-0114-X (2018).

8. Sepp, M. et al. The intellectual disability and schizophrenia associated transcription factor TCF4 Is regulated by neuronal activity and protein kinase A. J. Neurosci. 37, 10516-10527 (2017).

9. Ripke, S. et al. Biological insights from 108 schizophrenia-associated genetic loci. Nature 511, 421-427 (2014).

10. Stefansson, H. et al. Common variants conferring risk of schizophrenia. Nature 460, 744-747 (2009).

11. Doostparast Torshizi, A. et al. Deconvolution of transcriptional networks identifies TCF4 as a master regulator in schizophrenia. Sci. Adv. 5, 4139 (2019).

12. Kharbanda, M. et al. Partial deletion of TCF4 in three generation family with non-syndromic intellectual disability, without features of Pitt-Hopkins syndrome. Eur. J. Med. Genet. 59, 310-314 (2016).

13. Wray, N. R. et al. Genome-wide association analyses identify 44 risk variants and refine the genetic architecture of major depression. Nat. Genet. 50, 668 (2018).

14. Wieben, E. D. et al. A common trinucleotide repeat expansion within the transcription factor 4 (TCF4, E2-2) gene predicts Fuchs corneal dystrophy. PLoS ONE 7, e49083 (2012).

15. Del-Favero, J. et al. European combined analysis of the CTG18.1 and the ERDA1 CAG/CTG repeats in bipolar disorder. Eur. J. Hum. Genet. 10, 276-280 (2002).

16. Fautsch, M. P. et al. TCF4-mediated Fuchs endothelial corneal dystrophy: Insights into a common trinucleotide repeat-associated disease. Prog. Retinal Eye Res. https://doi.org/10.1016/j.preteyeres.2020.100883 (2020).

17. Ong Tone, S. et al. Fuchs endothelial corneal dystrophy: The vicious cycle of Fuchs pathogenesis. Prog. Retinal Eye Res. https://doi. org/10.1016/j.preteyeres.2020.100863 (2020).

18. Vieta, E. et al. Bipolar disorders. Nat. Rev. Dis. Primers 4, 1-16 (2018).

19. Foja, S., Luther, M., Hoffmann, K., Rupprecht, A. \& Gruenauer-Kloevekorn, C. CTG181 repeat expansion may reduce TCF4 gene expression in corneal endothelial cells of German patients with Fuchs' dystrophy. Graefes Arch. Clin. Exp. Ophthalmol. 255, 1621-1631 (2017).

20. Okumura, N. et al. Effect of trinucleotide repeat expansion on the expression of TCF4 mRNA in Fuchs' endothelial corneal dystrophy. Investig. Ophthalmol. Vis. Sci. 60, 779-786 (2019). 
21. Mootha, V. V. et al. TCF4 triplet repeat expansion and nuclear RNA foci in Fuchs' endothelial corneal dystrophy. Investig. Ophthalmol. Vis. Sci. 56, 2003-2011 (2015).

22. Ołdak, M. et al. Fuchs endothelial corneal dystrophy: Strong association with rs613872 not paralleled by changes in corneal endothelial TCF4 mRNA level. Biomed. Res. Int. 2015, 640234 (2015).

23. Ward, M. C. \& Gilad, Y. Human genomics: Cracking the regulatory code. Nature 550, 190-191 (2017).

24. Juven-Gershon, T., Hsu, J.-Y., Theisen, J. W. M. \& Kadonaga, J. T. The RNA polymerase II core promoter-The gateway to transcription. Curr. Opin. Cell Biol. 20, 253-259 (2008).

25. Brandenberger, R. et al. Transcriptome characterization elucidates signaling networks that control human ES cell growth and differentiation. Nat. Biotechnol. 22, 707-716 (2004).

26. Suzuki, Y. et al. Large-scale collection and characterization of promoters of human and mouse genes. In Silico Biol. 4, 429-444 (2004).

27. Nikitina, A. S. et al. Dataset on transcriptome profiling of corneal endothelium from patients with Fuchs endothelial corneal dystrophy. Data Brief 25, 104047 (2019).

28. Chu, Y. et al. Analyzing pre-symptomatic tissue to gain insights into the molecular and mechanistic origins of late-onset degenerative trinucleotide repeat disease. Nucleic Acids Res. 48, 6740-6758 (2020).

29. Paulson, H. Repeat expansion diseases. Handb. Clin. Neurol. 147, 105-123 (2018).

30. Soliman, A. Z., Xing, C., Radwan, S. H., Gong, X. \& Mootha, V. V. Correlation of severity of Fuchs endothelial corneal dystrophy with triplet repeat expansion in TCF4. JAMA Ophthalmol. 133, 1386-1391 (2015).

31. Rohilla, K. J. \& Gagnon, K. T. RNA biology of disease-associated microsatellite repeat expansions. Acta Neuropathol. Commun. https://doi.org/10.1186/s40478-017-0468-y (2017).

32. Du, J. et al. RNA toxicity and missplicing in the common eye disease Fuchs endothelial corneal dystrophy. J. Biol. Chem. 290, 5979-5990 (2015).

33. Rong, Z., Hu, J., Corey, D. R. \& Mootha, V. V. Quantitative studies of muscleblind proteins and their interaction with TCF4 RNA foci support involvement in the mechanism of Fuchs' dystrophy. Investig. Ophthalmol. Vis. Sci. 60, 3980-3991 (2019).

34. Hu, J. et al. Oligonucleotides targeting TCF4 triplet repeat expansion inhibit RNA foci and mis-splicing in Fuchs' dystrophy. Hum. Mol. Genet. https://doi.org/10.1093/hmg/ddy018 (2018).

35. Zarouchlioti, C. et al. Antisense therapy for a common corneal dystrophy ameliorates TCF4 repeat expansion-mediated toxicity. Am. J. Hum. Genet. https://doi.org/10.1016/j.ajhg.2018.02.010 (2018).

36. Nelson, D. L., Orr, H. T. \& Warren, S. T. The unstable repeats-Three evolving faces of neurological disease. Neuron $77,825-843$ (2013).

37. Salcedo-Arellano, M. J., Dufour, B., McLennan, Y., Martinez-Cerdeno, V. \& Hagerman, R. Fragile X syndrome and associated disorders: Clinical aspects and pathology. Neurobiol. Dis. 136, 104740 (2020).

38. Metsu, S. et al. FRA2A is a CGG repeat expansion associated with silencing of AFF3. PLoS Genet. 10, e1004242 (2014).

39. Chutake, Y. K., Lam, C., Costello, W. N., Anderson, M. \& Bidichandani, S. I. Epigenetic promoter silencing in Friedreich ataxia is dependent on repeat length. Ann. Neurol. 76, 522-528 (2014).

40. Gijselinck, I. et al. The C9orf72 repeat size correlates with onset age of disease, DNA methylation and transcriptional downregulation of the promoter. Mol. Psychiatry 21, 1112-1124 (2016).

41. Esvald, E.-E. et al. CREB family transcription factors are major mediators of BDNF transcriptional autoregulation in cortical neurons. J. Neurosci. 40, 1405-1426 (2020).

42. Lizio, M. et al. Gateways to the FANTOM5 promoter level mammalian expression atlas. Genome Biol. 16, 22 (2015).

\section{Acknowledgements}

We thank the 'TUT Institutional Development Program for 2016-2022' Graduate School in Clinical Medicine, which received funding from the European Regional Development Fund under program ASTRA 20142020.4.01.16-0032 in Estonia. The authors would also like to thank Laura Tamberg, Anastassia Šubina and Mari Maria Palgi for critical reading of the manuscript and Epp Väli for technical assistance.

\section{Author contributions}

A.S.: conceptualization, writing, experimentation, bioinformatics; K.L.: conceptualization, writing, experimentation; J.T.: conceptualization, writing, bioinformatics; K.N.: conceptualization, writing, supervision; M.S.: conceptualization, writing supervision, funding acquisition; T.T.: conceptualization, writing, supervision, funding acquisition.

\section{Funding}

This project was supported by Estonian Research Council (institutional research funding IUT19-18 and grant PRG805), European Union through the European Regional Development Fund (Project No. 2014-2020.4.01.150012), H2020-MSCA-RISE-2016 (Grant EU734791), Pitt Hopkins Research Foundation (Grants No. 8 and No. 21) and Million Dollar Bike Ride Pilot Grant Program for Rare Disease Research at UPenn Orphan Disease Center (Grants MDBR-16-122-PHP and MDBR-17-127-Pitt Hopkins). The funding sources were not involved in study design, analysis and interpretation of data, writing of the report and in the decision to submit the article for publication.

\section{Competing interests}

The authors declare no competing interests.

\section{Additional information}

Supplementary information is available for this paper at https://doi.org/10.1038/s41598-020-75437-3.

Correspondence and requests for materials should be addressed to T.T.

Reprints and permissions information is available at www.nature.com/reprints.

Publisher's note Springer Nature remains neutral with regard to jurisdictional claims in published maps and institutional affiliations. 
(c) (i) Open Access This article is licensed under a Creative Commons Attribution 4.0 International cc) License, which permits use, sharing, adaptation, distribution and reproduction in any medium or format, as long as you give appropriate credit to the original author(s) and the source, provide a link to the Creative Commons licence, and indicate if changes were made. The images or other third party material in this article are included in the article's Creative Commons licence, unless indicated otherwise in a credit line to the material. If material is not included in the article's Creative Commons licence and your intended use is not permitted by statutory regulation or exceeds the permitted use, you will need to obtain permission directly from the copyright holder. To view a copy of this licence, visit http://creativecommons.org/licenses/by/4.0/.

(C) The Author(s) 2020 